\title{
Absorption Spectra and Electronic Structure of Gallic Acid in Water at Different pH: Experimental Data and Theoretical Cluster Models
}

\author{
Natalia V. Karimova ${ }^{1}$, Man Luo ${ }^{2}$, Izaac Sit ${ }^{3}$, Vicki H. Grassian*2,3, R. Benny Gerber*1,4
}

${ }^{1}$ Department of Chemistry, University of California, Irvine, CA 92697, USA

${ }^{2}$ Department of Chemistry and Biochemistry, University of California, San Diego, CA 92093, USA

${ }^{3}$ Department of Nanoengineering and Scripps Institution of Oceanography, University of California, San Diego, CA 92093, USA

${ }^{4}$ Institute of Chemistry and Fritz Haber Research Center, Hebrew University of Jerusalem, Jerusalem 91904, Israel

\begin{tabular}{|c|c|c|}
\hline Name & Description & Pages \\
\hline Figure S1 & $\begin{array}{l}\text { Structure examples of the cluster-models: (a) neutral } \\
\mathrm{GA} \cdot\left(\mathrm{H}_{2} \mathrm{O}\right)_{4}\left(\mathrm{H}_{2} \mathrm{O}\right)_{1} \text { and (b) ionized } \mathrm{GA}^{-} \cdot\left(\mathrm{H}_{2} \mathrm{O}\right)_{4}\left(\mathrm{H}_{2} \mathrm{O}\right)_{1}\end{array}$ & S2 \\
\hline Figure S2 & $\begin{array}{l}\text { Structure examples of the cluster-models: (a) neutral } \\
\mathrm{GA} \cdot\left(\mathrm{H}_{2} \mathrm{O}\right)_{4}\left(\mathrm{H}_{2} \mathrm{O}\right)_{1} \text { and (b) ionized } \mathrm{GA}^{-} \cdot\left(\mathrm{H}_{2} \mathrm{O}\right)_{4}\left(\mathrm{H}_{2} \mathrm{O}\right)_{2}\end{array}$ & S3 \\
\hline Figure S3 & $\begin{array}{l}\text { Experimental optical absorption spectrum of gallic acid at } \mathrm{pH}=2 \text { (red line) and } \\
\text { theoretical (black line) optical spectra of isolated neutral gallic acid calculated } \\
\text { using ADC(3), ADC(2) and B3LYP methods together with 6-311+G* and 6- } \\
311++\mathrm{G}^{* *} \text { basis sets, with and without polarized continuum model (PCM). }\end{array}$ & S4 \\
\hline Figure S4 & $\begin{array}{l}\text { Calculated optical absorption spectra for neutral } \mathrm{GA} \cdot\left(\mathrm{H}_{2} \mathrm{O}\right) \mathrm{n} \text { and ionized } \mathrm{GA}^{-} \\
\cdot\left(\mathrm{H}_{2} \mathrm{O}\right) \text { n clusters using B3LYP/6-311++蛙* (gas phase) method. }\end{array}$ & S5 \\
\hline Table S1 & $\begin{array}{l}\text { Calculated excited states characteristics such as position }(\mathrm{eV} \text { and } \mathrm{nm} \text { ) and } \\
\text { oscillator strength(f). Method is B3LYP/6-311++G** (gas phase). System is } \\
\text { neutral GA. }\left(\mathrm{H}_{2} \mathrm{O}\right) \mathrm{n}\end{array}$ & S6-S7 \\
\hline Table S2 & $\begin{array}{l}\text { Calculated excited states characteristics such as position }(\mathrm{eV} \text { and } \mathrm{nm}) \text { and } \\
\text { oscillator strength(f). Method is B3LYP/6-311++G** (with C-PCM). System is } \\
\text { ionized } \mathrm{GA}^{-} \cdot\left(\mathrm{H}_{2} \mathrm{O}\right) \text { n. }\end{array}$ & S7-S11 \\
\hline Figure S5 & $\begin{array}{l}\text { Calculated optical absorption spectra for neutral GA }\left(\mathrm{H}_{2} \mathrm{O}\right) \text { n and ionized } \mathrm{GA}^{-} \\
\cdot\left(\mathrm{H}_{2} \mathrm{O}\right) \text { n clusters using B3LYP/6-311++G** (with C-PCM) method. }\end{array}$ & S12 \\
\hline Figure S6 & $\begin{array}{l}\text { Calculated optical absorption spectra for neutral } \mathrm{GA} \cdot\left(\mathrm{H}_{2} \mathrm{O}\right) \mathrm{n} \cdot\left(\mathrm{H}_{2} \mathrm{O}\right) \mathrm{m} \text { and ionized } \\
\mathrm{GA}^{-} \cdot\left(\mathrm{H}_{2} \mathrm{O}\right) \mathrm{n} \cdot\left(\mathrm{H}_{2} \mathrm{O}\right) \text { m clusters using B3LYP/6-311++G** (with C-PCM) method. }\end{array}$ & S13 \\
\hline
\end{tabular}




\begin{tabular}{|c|l|c|}
\hline Table S3 & $\begin{array}{l}\text { Calculated excited states characteristics such as position }(\mathrm{eV} \text { and } \mathrm{nm}) \text { and } \\
\text { oscillator strength(f). Method is B3LYP/6-311++G** }(\text { with C-PCM). System is } \\
\text { neutral } \mathrm{GA} \cdot\left(\mathrm{H}_{2} \mathrm{O}\right) \mathrm{n} \cdot\left(\mathrm{H}_{2} \mathrm{O}\right) \mathrm{m}\end{array}$ & S13-S15 \\
\hline Table S4 & $\begin{array}{l}\text { Calculated excited states characteristics such as position }(\mathrm{eV} \text { and } \mathrm{nm}) \text { and } \\
\text { oscillator strength(f). Method is B3LYP/6-311++G** (with C-PCM). System is } \\
\text { ionized } \mathrm{GA}^{-} \cdot\left(\mathrm{H}_{2} \mathrm{O}\right) \mathrm{n} \cdot\left(\mathrm{H}_{2} \mathrm{O}\right) \mathrm{m}\end{array}$ & $\mathrm{S} 16-\mathrm{S} 18$ \\
\hline Table S5 & $\begin{array}{l}\text { Experimental and Theoretical peak positions }(\lambda, \mathrm{nm}) \text { and oscillator strength }(\mathrm{f}) \text { of } \\
\text { optical absorption spectra of } \mathrm{GA} \text { at different } \mathrm{pH} .\end{array}$ & $\mathrm{S} 19$ \\
\hline
\end{tabular}

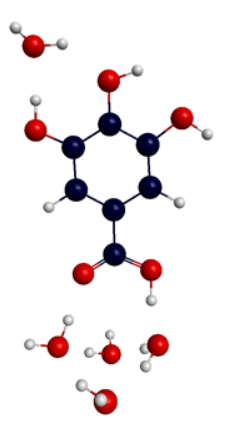

V1

$0 \mathrm{kcal} / \mathrm{mol}$

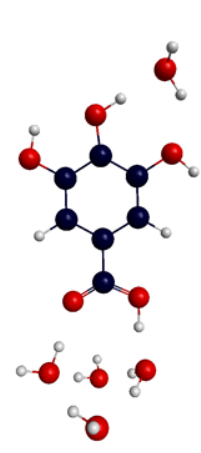

V2 $-1 \mathrm{kcal} / \mathrm{mol}$

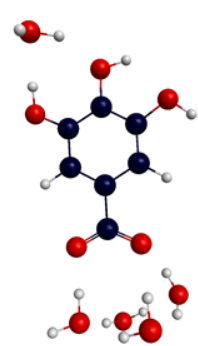

V1

$0 \mathrm{kcal} / \mathrm{mol}$

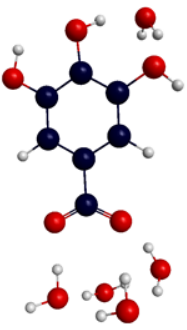

V3

$-1.6 \mathrm{kcal} / \mathrm{mol}$

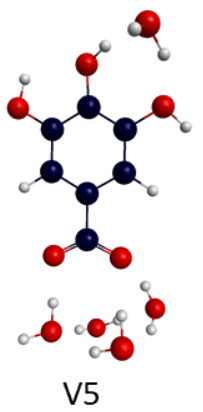

$-1.7 \mathrm{kcal} / \mathrm{mol}$

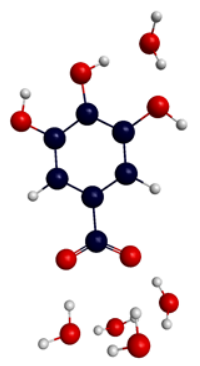

V2

$-0.9 \mathrm{kcal} / \mathrm{mol}$

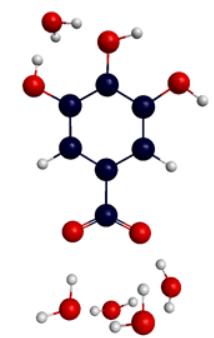

V4

$-0.01 \mathrm{kcal} / \mathrm{mol}$



$0.03 \mathrm{kcal} / \mathrm{mol}$

Figure S1. Structure examples of the cluster-models: (a) neutral GA· $\left(\mathrm{H}_{2} \mathrm{O}\right)_{4}\left(\mathrm{H}_{2} \mathrm{O}\right)_{1}$ and (b) ionized $\mathrm{GA}^{-} \cdot\left(\mathrm{H}_{2} \mathrm{O}\right)_{4}\left(\mathrm{H}_{2} \mathrm{O}\right)_{1}$. 


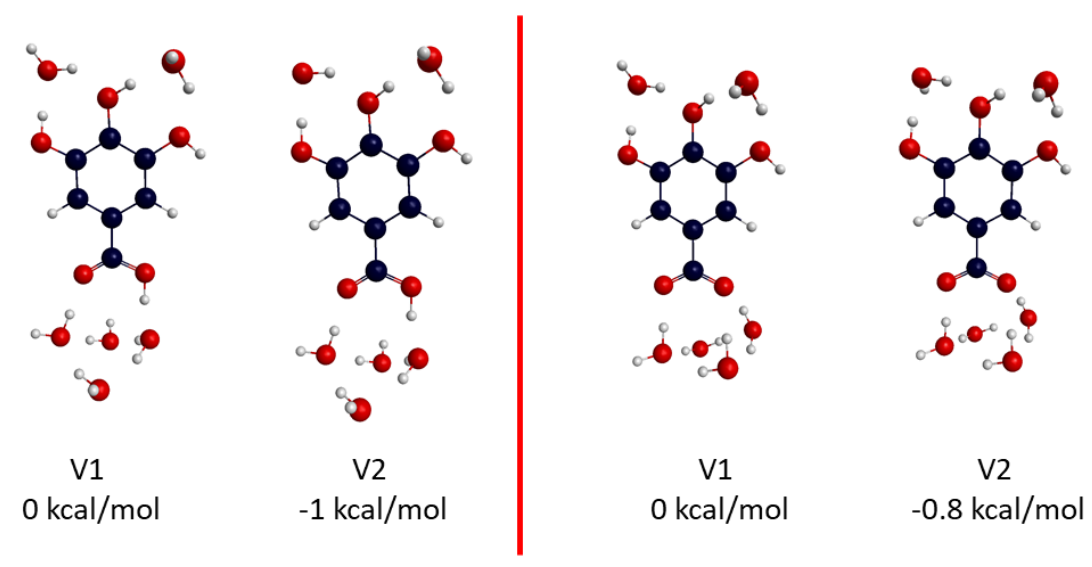

Figure S2. Structure examples of the cluster-models: (a) neutral $\mathrm{GA} \cdot\left(\mathrm{H}_{2} \mathrm{O}\right)_{4}\left(\mathrm{H}_{2} \mathrm{O}\right)_{1}$ and (b) ionized $\mathrm{GA}^{-} \cdot\left(\mathrm{H}_{2} \mathrm{O}\right)_{4}\left(\mathrm{H}_{2} \mathrm{O}\right)_{2}$. 

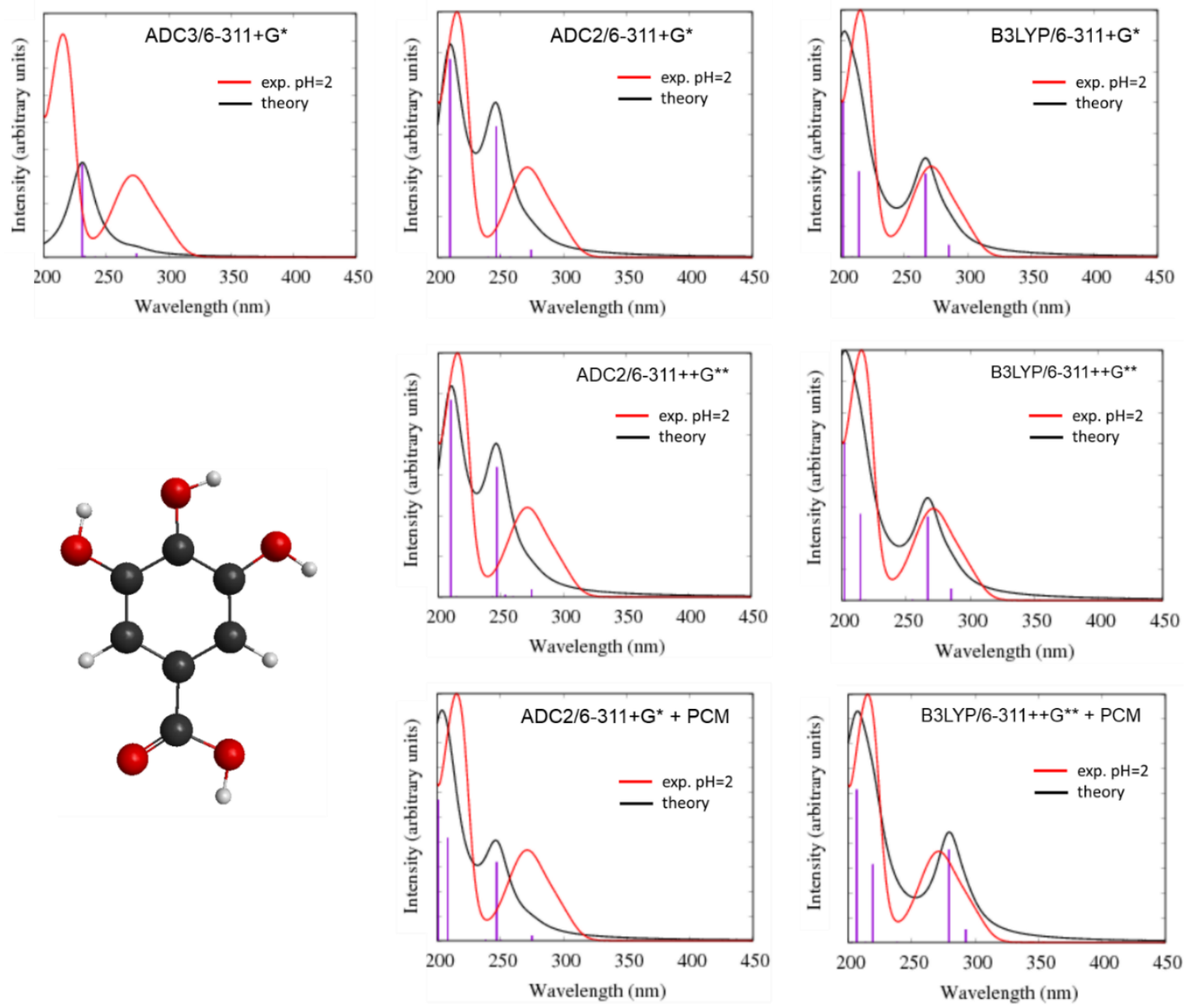

Figure S3. Experimental optical absorption spectrum of gallic acid at $\mathrm{pH}=2$ (red line) and theoretical (black line) optical spectra of isolated neutral gallic acid calculated using ADC(3), ADC(2) and B3LYP methods together with 6-311+G* and 6-311++G** basis sets, with and without polarized continuum model (PCM). 

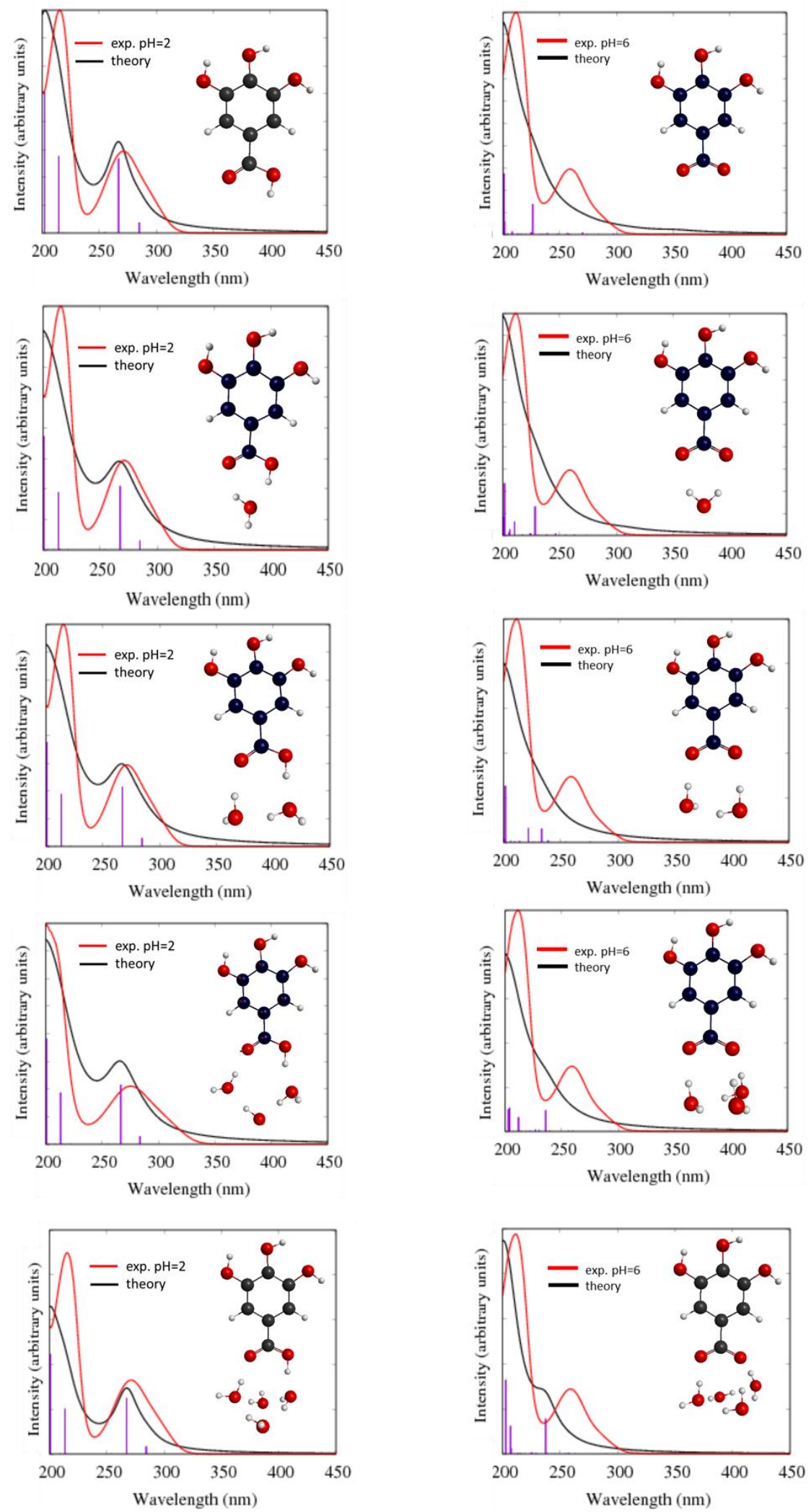

Figure S4. Calculated optical absorption spectra for neutral $\mathrm{GA} \cdot\left(\mathrm{H}_{2} \mathrm{O}\right) \mathrm{n}$ and ionized $\mathrm{GA}^{-} \cdot\left(\mathrm{H}_{2} \mathrm{O}\right)$ n clusters using B3LYP/6-311++G** (gas phase) method. 
Table S1. Calculated excited states characteristics such as position (eV and $\mathrm{nm}$ ) and oscillator strength(f). Method is B3LYP/6-311++G** (gas phase). System is neutral GA $\left(\mathrm{H}_{2} \mathrm{O}\right) \mathrm{n}$

\begin{tabular}{|c|c|c|c|c|}
\hline & \#Ex.State & $\mathrm{eV}$ & $\mathrm{nm}$ & $f$ \\
\hline \multirow[t]{12}{*}{$n=0$} & 1 & 4.3489 & 285 & 0.029862 \\
\hline & 2 & 4.6422 & 267 & 0.210309 \\
\hline & 3 & 4.8595 & 255 & 0.001489 \\
\hline & 4 & 4.8962 & 253 & 3.61E-05 \\
\hline & 5 & 5.0076 & 248 & $4.86 \mathrm{E}-05$ \\
\hline & 6 & 5.5004 & 225 & $3.03 E-05$ \\
\hline & 7 & 5.6817 & 218 & 0.000181 \\
\hline & 8 & 5.7774 & 215 & 0.217018 \\
\hline & 9 & 5.8137 & 213 & 9.89E-05 \\
\hline & 10 & 5.9769 & 207 & 8.57E-07 \\
\hline & 11 & 6.1358 & 202 & 0.398765 \\
\hline & \#Ex.State & $\mathrm{eV}$ & $\mathrm{nm}$ & $f$ \\
\hline \multirow[t]{11}{*}{$n=1$} & 1 & 4.3521 & 285 & 0.033252 \\
\hline & 2 & 4.636 & 267 & 0.229718 \\
\hline & 3 & 4.8743 & 254 & 0.001139 \\
\hline & 4 & 5.0305 & 246 & 0.0001 \\
\hline & 5 & 5.0518 & 245 & 4.06E-05 \\
\hline & 6 & 5.5932 & 222 & 0.000133 \\
\hline & 7 & 5.773 & 215 & 0.001627 \\
\hline & 8 & 5.8105 & 213 & 0.207656 \\
\hline & 9 & 5.9123 & 210 & 0.000789 \\
\hline & 10 & 6.0786 & 204 & 0.000677 \\
\hline & \#Ex.State & $\mathrm{eV}$ & $\mathrm{nm}$ & $f$ \\
\hline \multirow[t]{10}{*}{$n=2$} & 1 & 4.355 & 285 & 0.033049 \\
\hline & 2 & 4.6376 & 267 & 0.235831 \\
\hline & 3 & 4.8632 & 255 & 0.001117 \\
\hline & 4 & 5.0179 & 247 & $8.58 \mathrm{E}-05$ \\
\hline & 5 & 5.1056 & 243 & 8.79E-05 \\
\hline & 6 & 5.5903 & 222 & 0.000139 \\
\hline & 7 & 5.7694 & 215 & 0.000278 \\
\hline & 8 & 5.8104 & 213 & 0.206388 \\
\hline & 9 & 5.9623 & 208 & 0.000225 \\
\hline & 10 & 6.1272 & 202 & 0.003147 \\
\hline
\end{tabular}




\begin{tabular}{|c|c|c|c|c|}
\hline & \#Ex.State & $\mathrm{eV}$ & $\mathrm{nm}$ & $f$ \\
\hline \multirow[t]{11}{*}{$n=3$} & 1 & 4.3713 & 284 & 0.032529 \\
\hline & 2 & 4.653 & 266 & 0.23685 \\
\hline & 3 & 4.8562 & 255 & 0.001118 \\
\hline & 4 & 5.0124 & 247 & $8.65 E-05$ \\
\hline & 5 & 5.0787 & 244 & 4.17E-05 \\
\hline & 6 & 5.5857 & 222 & 0.000138 \\
\hline & 7 & 5.7647 & 215 & 0.000542 \\
\hline & 8 & 5.8139 & 213 & 0.205652 \\
\hline & 9 & 5.951 & 208 & 0.000516 \\
\hline & 10 & 6.1159 & 203 & 0.000614 \\
\hline & \#Ex.State & $\mathrm{eV}$ & $\mathrm{nm}$ & $f$ \\
\hline \multirow[t]{11}{*}{$n=4$} & 1 & 4.3602 & 284 & 0.033277 \\
\hline & 2 & 4.6367 & 267 & 0.244944 \\
\hline & 3 & 4.878 & 254 & 0.001075 \\
\hline & 4 & 5.0389 & 246 & 7.05E-05 \\
\hline & 5 & 5.184 & 239 & $5.41 \mathrm{E}-05$ \\
\hline & 6 & 5.6144 & 221 & 9.23E-05 \\
\hline & 7 & 5.7615 & 215 & $1.44 \mathrm{E}-05$ \\
\hline & 8 & 5.7834 & 214 & 0.000138 \\
\hline & 9 & 5.8087 & 213 & 0.198652 \\
\hline & 10 & 5.9244 & 209 & 0.000261 \\
\hline & 11 & 6.0766 & 204 & 4.64E-05 \\
\hline
\end{tabular}

Table S2. Calculated excited states characteristics such as position ( $\mathrm{eV}$ and $\mathrm{nm}$ ) and oscillator strength(f). Method is B3LYP/6-311++G** (with C-PCM). System is ionized $\mathrm{GA}^{-} \cdot\left(\mathrm{H}_{2} \mathrm{O}\right)$ n.

\begin{tabular}{|r|r|r|r|r|}
\hline & \#Ex.State & \multicolumn{1}{c|}{$\mathrm{eV}$} & $\mathrm{nm}$ & $\mathrm{f}$ \\
\hline $\mathrm{n}=0$ & 1 & 3.24 & 383 & 0.000181 \\
\hline & 2 & 3.5077 & 353 & 0.006056 \\
\hline & 3 & 3.623 & 342 & $2.59 \mathrm{E}-06$ \\
\hline & 4 & 3.7864 & 327 & $5.51 \mathrm{E}-07$ \\
\hline & 5 & 3.9016 & 318 & 0.000236 \\
\hline & 6 & 3.9106 & 317 & 0.000961 \\
\hline & 7 & 4.0673 & 305 & $3.42 \mathrm{E}-08$ \\
\hline & 8 & 4.1645 & 298 & 0.000121 \\
\hline & 9 & 4.1715 & 297 & 0.004656 \\
\hline & 10 & 4.2854 & 289 & $7.09 \mathrm{E}-05$ \\
\hline & 11 & 4.3678 & 284 & 0.000174 \\
\hline & 12 & 4.527 & 274 & 0.00171 \\
\hline
\end{tabular}




\begin{tabular}{|c|c|c|c|c|}
\hline & 13 & 4.5763 & 271 & $1.80 \mathrm{E}-07$ \\
\hline & 14 & 4.589 & 270 & 0.009903 \\
\hline & 15 & 4.638 & 267 & 5.46E-05 \\
\hline & 16 & 4.7983 & 258 & 0.002286 \\
\hline & 17 & 4.826 & 257 & 0.00756 \\
\hline & 18 & 4.8518 & 256 & 0.000152 \\
\hline & 19 & 4.9156 & 252 & $6.13 \mathrm{E}-07$ \\
\hline & 20 & 4.9951 & 248 & 0.002109 \\
\hline & 21 & 5.1875 & 239 & 0.007157 \\
\hline & 22 & 5.2588 & 236 & 0.000358 \\
\hline & 23 & 5.3304 & 233 & 0.001088 \\
\hline & 24 & 5.4824 & 226 & 0.151331 \\
\hline & 25 & 5.5094 & 225 & 0.000476 \\
\hline & 26 & 5.5216 & 225 & 0.009259 \\
\hline & 27 & 5.5674 & 223 & $3.22 \mathrm{E}-05$ \\
\hline & 28 & 5.5892 & 222 & $1.61 \mathrm{E}-06$ \\
\hline & 29 & 5.6454 & 220 & 0.001311 \\
\hline & 30 & 5.7455 & 216 & 0.003331 \\
\hline & 31 & 5.8012 & 214 & 0.000352 \\
\hline & 32 & 5.8298 & 213 & 0.003796 \\
\hline & 33 & 5.9566 & 208 & 0.013959 \\
\hline & 34 & 6.0697 & 204 & 0.003734 \\
\hline & \#Ex.State & $\mathrm{eV}$ & $\mathrm{nm}$ & $f$ \\
\hline$n=1$ & 1 & 3.861 & 321 & 0.00011 \\
\hline & 2 & 4.0356 & 307 & 0.00526 \\
\hline & 3 & 4.0406 & 307 & 0.000844 \\
\hline & 4 & 4.103 & 302 & 0.000109 \\
\hline & 5 & 4.1381 & 300 & $1.81 \mathrm{E}-07$ \\
\hline & 6 & 4.3492 & 285 & $2.42 \mathrm{E}-05$ \\
\hline & 7 & 4.3697 & 284 & 0.00013 \\
\hline & 8 & 4.5336 & 273 & 0.000195 \\
\hline & 9 & 4.711 & 263 & 0.004135 \\
\hline & 10 & 4.7451 & 261 & $8.42 \mathrm{E}-07$ \\
\hline & 11 & 4.8254 & 257 & 3.73E-05 \\
\hline & 12 & 4.8473 & 256 & 0.001048 \\
\hline & 13 & 4.8481 & 256 & 3.96E-05 \\
\hline & 14 & 4.8659 & 255 & 0.000147 \\
\hline & 15 & 4.9967 & 248 & 4.10E-07 \\
\hline & 16 & 5.0275 & 247 & 0.00918 \\
\hline & 17 & 5.0582 & 245 & 0.000226 \\
\hline
\end{tabular}




\begin{tabular}{|c|c|c|c|c|}
\hline & 18 & 5.1637 & 240 & 0.001652 \\
\hline & 19 & 5.2045 & 238 & 0.003428 \\
\hline & 20 & 5.3452 & 232 & 0.000789 \\
\hline & 21 & 5.3824 & 230 & 0.000333 \\
\hline & 22 & 5.426 & 228 & 0.144051 \\
\hline & 23 & 5.4619 & 227 & 7.80E-05 \\
\hline & 24 & 5.5154 & 225 & 0.000137 \\
\hline & 25 & 5.5281 & 224 & 0.01128 \\
\hline & 26 & 5.6983 & 218 & 0.002842 \\
\hline & 27 & 5.7155 & 217 & 0.000358 \\
\hline & 28 & 5.8975 & 210 & 0.067794 \\
\hline & 29 & 6.003 & 207 & 2.92E-06 \\
\hline & \#Ex.State & $\mathrm{eV}$ & $\mathrm{nm}$ & $f$ \\
\hline$n=2$ & 1 & 4.1335 & 300 & 0.00099 \\
\hline & 2 & 4.1876 & 296 & 0.000417 \\
\hline & 3 & 4.2774 & 290 & 5.99E-06 \\
\hline & 4 & 4.3039 & 288 & 4.95E-05 \\
\hline & 5 & 4.4935 & 276 & 0.004158 \\
\hline & 6 & 4.6234 & 268 & 4.93E-05 \\
\hline & 7 & 4.6328 & 268 & 0.000109 \\
\hline & 8 & 4.8648 & 255 & 0.000202 \\
\hline & 9 & 4.8743 & 254 & 0.000542 \\
\hline & 10 & 4.8786 & 254 & 0.00084 \\
\hline & 11 & 4.9067 & 253 & 4.40E-07 \\
\hline & 12 & 5.0073 & 248 & 1.06E-05 \\
\hline & 13 & 5.1218 & 242 & 0.000109 \\
\hline & 14 & 5.1707 & 240 & 0.001706 \\
\hline & 15 & 5.1853 & 239 & 0.01164 \\
\hline & 16 & 5.3108 & 233 & 0.085253 \\
\hline & 17 & 5.3152 & 233 & 0.001445 \\
\hline & 18 & 5.3643 & 231 & 0.000661 \\
\hline & 19 & 5.4507 & 227 & 0.002246 \\
\hline & 20 & 5.4962 & 226 & 0.000578 \\
\hline & 21 & 5.5044 & 225 & 0.000797 \\
\hline & 22 & 5.5872 & 222 & 0.004177 \\
\hline & 23 & 5.5892 & 222 & 0.088464 \\
\hline & 24 & 5.6638 & 219 & 0.000121 \\
\hline & 25 & 5.7305 & 216 & $1.64 \mathrm{E}-05$ \\
\hline & 26 & 5.7921 & 214 & 0.00908 \\
\hline & 27 & 5.9186 & 209 & 0.005884 \\
\hline
\end{tabular}




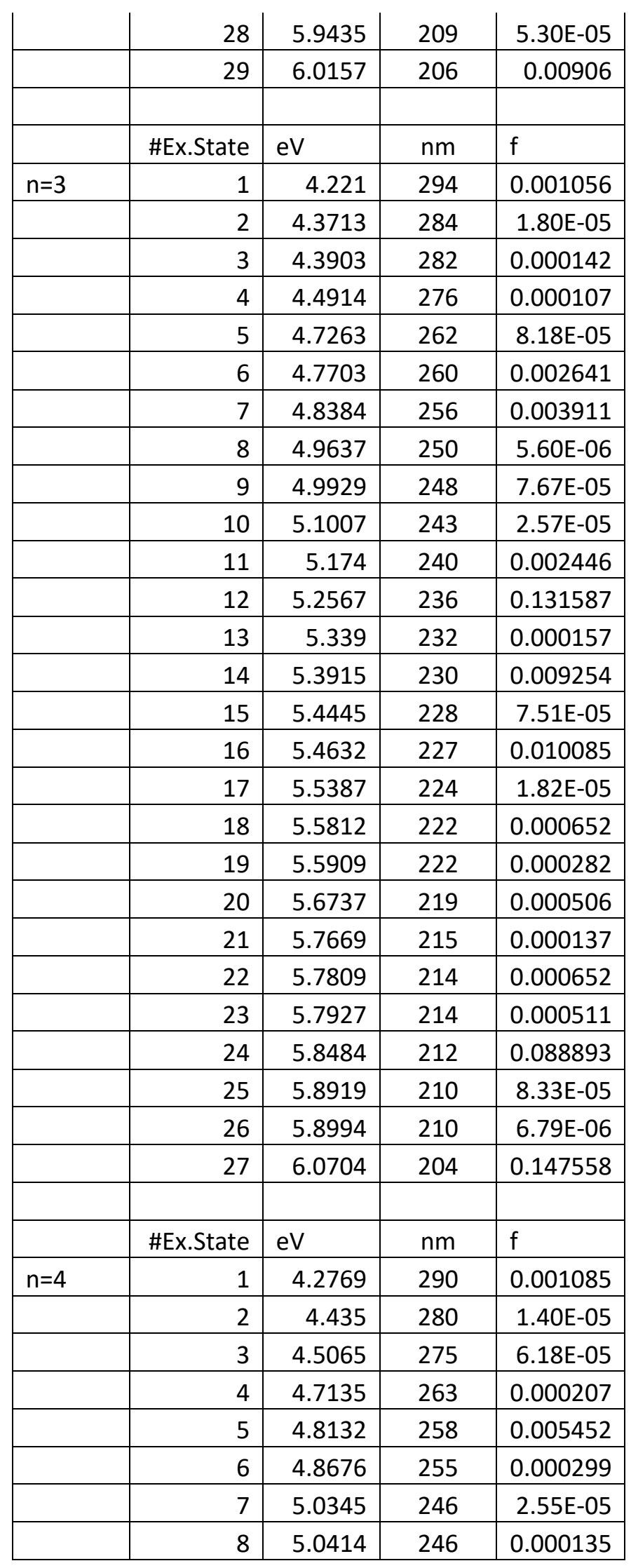




\begin{tabular}{|r|r|r|r|r|} 
& 9 & 5.1287 & 242 & $5.08 \mathrm{E}-05$ \\
\hline & 10 & 5.1742 & 240 & $4.40 \mathrm{E}-05$ \\
\hline & 11 & 5.1987 & 238 & 0.002163 \\
\hline & 12 & 5.2204 & 237 & 0.153309 \\
\hline & 13 & 5.4071 & 229 & 0.005557 \\
\hline & 14 & 5.4274 & 228 & 0.000113 \\
\hline & 15 & 5.4962 & 226 & 0.005981 \\
\hline & 16 & 5.5185 & 225 & 0.001878 \\
\hline & 17 & 5.6086 & 221 & $5.72 \mathrm{E}-05$ \\
\hline & 18 & 5.6481 & 220 & 0.000321 \\
\hline & 19 & 5.7349 & 216 & 0.002336 \\
\hline & 20 & 5.7374 & 216 & 0.000373 \\
\hline & 21 & 5.787 & 214 & 0.002217 \\
\hline & 22 & 5.8181 & 213 & 0.000305 \\
\hline & 23 & 5.8308 & 213 & 0.000157 \\
\hline & 24 & 5.8663 & 211 & 0.000101 \\
\hline & 25 & 5.8878 & 211 & 0.000358 \\
\hline & 26 & 5.9745 & 208 & 0.024714 \\
\hline & 27 & 5.9916 & 207 & 0.12225 \\
\hline & 28 & 5.9928 & 207 & 0.009288 \\
\hline & 6.1087 & 203 & 0.322902 \\
\hline
\end{tabular}





Figure S5. Calculated optical absorption spectra for neutral $\mathrm{GA} \cdot\left(\mathrm{H}_{2} \mathrm{O}\right) \mathrm{n}$ and ionized $\mathrm{GA}^{-} \cdot\left(\mathrm{H}_{2} \mathrm{O}\right) n$ clusters using B3LYP/6-311++G** (with C-PCM) method. 



Figure S6. Calculated optical absorption spectra for neutral GA$\cdot\left(\mathrm{H}_{2} \mathrm{O}\right) \mathrm{n} \cdot\left(\mathrm{H}_{2} \mathrm{O}\right) \mathrm{m}$ and ionized $\mathrm{GA}^{-} \cdot\left(\mathrm{H}_{2} \mathrm{O}\right) \mathrm{n} \cdot\left(\mathrm{H}_{2} \mathrm{O}\right) \mathrm{m}$ clusters using B3LYP/6-311++G** (with C-PCM) method.

Table S3. Calculated excited states characteristics such as position ( $\mathrm{eV}$ and $\mathrm{nm}$ ) and oscillator strength(f). Method is B3LYP/6-311++G** (with C-PCM). System is neutral GA $\left(\mathrm{H}_{2} \mathrm{O}\right) n \cdot\left(\mathrm{H}_{2} \mathrm{O}\right) \mathrm{m}$

\begin{tabular}{|c|c|c|c|c|}
\hline $\mathrm{n}=0$ & \#Ex.State & $\mathrm{eV}$ & $\mathrm{nm}$ & $\mathrm{f}$ \\
\hline & 1 & 4.2353 & 293 & 0.041642 \\
\hline & 2 & 4.4362 & 279 & 0.293867 \\
\hline & 3 & 5.0709 & 244 & 0.000010 \\
\hline & 4 & 5.1952 & 239 & 0.001297 \\
\hline & 5 & 5.3851 & 230 & 0.000002 \\
\hline & 6 & 5.6521 & 219 & 0.247485 \\
\hline & 7 & 5.8001 & 214 & 0.000145 \\
\hline & 8 & 5.9091 & 210 & 0.000013 \\
\hline & 9 & 5.9986 & 207 & 0.483766 \\
\hline & 10 & 6.009 & 206 & 0.000098 \\
\hline
\end{tabular}




\begin{tabular}{|c|c|c|c|c|}
\hline & \#Ex.State & $\mathrm{eV}$ & $\mathrm{nm}$ & $f$ \\
\hline \multirow[t]{12}{*}{$n=1$} & 1 & 4.2297 & 293 & 0.041451 \\
\hline & 2 & 4.4262 & 280 & 0.310442 \\
\hline & 3 & 5.1211 & 242 & 0.000035 \\
\hline & 4 & 5.1817 & 239 & 0.001345 \\
\hline & 5 & 5.3711 & 231 & 0.000007 \\
\hline & 6 & 5.6479 & 220 & 0.241199 \\
\hline & 7 & 5.72 & 217 & 0.000081 \\
\hline & 8 & 5.8368 & 212 & 0.000103 \\
\hline & 9 & 5.981 & 207 & 0.000814 \\
\hline & 10 & 5.9967 & 207 & 0.498294 \\
\hline & 11 & 6.0854 & 204 & 0.001234 \\
\hline & \#Ex.State & $\mathrm{eV}$ & $\mathrm{nm}$ & $f$ \\
\hline \multirow[t]{12}{*}{$n=2$} & 1 & 4.2405 & 292 & 0.040503 \\
\hline & 2 & 4.4358 & 279 & 0.315689 \\
\hline & 3 & 5.1614 & 240 & 0.000097 \\
\hline & 4 & 5.171 & 240 & 0.001317 \\
\hline & 5 & 5.3569 & 231 & 0.000004 \\
\hline & 6 & 5.6477 & 220 & 0.234390 \\
\hline & 7 & 5.6596 & 219 & 0.005378 \\
\hline & 8 & 5.7772 & 215 & 0.000094 \\
\hline & 9 & 5.9619 & 208 & 0.001599 \\
\hline & 10 & 5.9932 & 207 & 0.502135 \\
\hline & 11 & 6.0646 & 204 & 0.001435 \\
\hline & \#Ex.State & $\mathrm{eV}$ & $\mathrm{nm}$ & $f$ \\
\hline \multirow[t]{12}{*}{$n=3$} & 1 & 4.2524 & 292 & 0.040199 \\
\hline & 2 & 4.4476 & 279 & 0.315740 \\
\hline & 3 & 5.1437 & 241 & 0.000000 \\
\hline & 4 & 5.1639 & 240 & 0.001367 \\
\hline & 5 & 5.3507 & 232 & 0.000006 \\
\hline & 6 & 5.5906 & 222 & 0.000051 \\
\hline & 7 & 5.6494 & 219 & 0.240013 \\
\hline & 8 & 5.7106 & 217 & 0.000098 \\
\hline & 9 & 5.9445 & 209 & 0.000297 \\
\hline & 10 & 5.9909 & 207 & 0.507538 \\
\hline & 11 & 6.0465 & 205 & 0.001038 \\
\hline & \#Ex.State & $\mathrm{eV}$ & $\mathrm{nm}$ & $f$ \\
\hline
\end{tabular}




\begin{tabular}{|c|c|c|c|c|}
\hline \multirow[t]{12}{*}{$n=4$} & 1 & 4.2556 & 291 & 0.043097 \\
\hline & 2 & 4.4524 & 278 & 0.317821 \\
\hline & 3 & 5.1473 & 241 & 0.001236 \\
\hline & 4 & 5.1962 & 239 & 0.000132 \\
\hline & 5 & 5.3438 & 232 & 0.000003 \\
\hline & 6 & 5.5119 & 225 & 0.000015 \\
\hline & 7 & 5.6463 & 220 & 0.244876 \\
\hline & 8 & 5.6544 & 219 & 0.000034 \\
\hline & 9 & 5.9408 & 209 & 0.000147 \\
\hline & 10 & 5.992 & 207 & 0.511172 \\
\hline & 11 & 6.0606 & 205 & 0.000986 \\
\hline & \#Ex.State & $\mathrm{eV}$ & $\mathrm{nm}$ & $f$ \\
\hline \multirow[t]{12}{*}{$n=4 m=1$} & 1 & 4.2335 & 293 & 0.072106 \\
\hline & 2 & 4.4088 & 281 & 0.316204 \\
\hline & 3 & 5.1065 & 243 & 0.001080 \\
\hline & 4 & 5.206 & 238 & 0.000090 \\
\hline & 5 & 5.3542 & 232 & 0.000012 \\
\hline & 6 & 5.4255 & 229 & 0.000008 \\
\hline & 7 & 5.5717 & 223 & 0.242173 \\
\hline & 8 & 5.6449 & 220 & 0.000087 \\
\hline & 9 & 5.7965 & 214 & 0.000419 \\
\hline & 10 & 5.9742 & 208 & 0.500307 \\
\hline & 11 & 6.0087 & 206 & 0.005724 \\
\hline & \#Ex.State & $\mathrm{eV}$ & $\mathrm{nm}$ & $f$ \\
\hline \multirow[t]{12}{*}{$n=4 m=2$} & 1 & 4.148 & 299 & 0.065062 \\
\hline & 2 & 4.4563 & 278 & 0.296515 \\
\hline & 3 & 5.119 & 242 & 0.001220 \\
\hline & 4 & 5.1872 & 239 & 0.000181 \\
\hline & 5 & 5.31 & 233 & 0.000581 \\
\hline & 6 & 5.4442 & 228 & 0.000023 \\
\hline & 7 & 5.5879 & 222 & 0.273914 \\
\hline & 8 & 5.6433 & 220 & 0.000041 \\
\hline & 9 & 5.7252 & 217 & 0.003250 \\
\hline & 10 & 5.8818 & 211 & 0.448011 \\
\hline & 11 & 5.942 & 209 & 0.010520 \\
\hline & 12 & 6.0601 & 205 & 0.002455 \\
\hline
\end{tabular}


Table S4. Calculated excited states characteristics such as position (eV and $\mathrm{nm}$ ) and oscillator strength(f). Method is B3LYP/6-311++G** (with C-PCM). System is ionized $\mathrm{GA}^{-} \cdot\left(\mathrm{H}_{2} \mathrm{O}\right) \mathrm{n} \cdot\left(\mathrm{H}_{2} \mathrm{O}\right) \mathrm{m}$

\begin{tabular}{|c|c|c|c|c|}
\hline & \#Ex.State & $\mathrm{eV}$ & $\mathrm{nm}$ & $f$ \\
\hline \multirow[t]{19}{*}{$n=0$} & 1 & 4.7421 & 261 & 0.000001 \\
\hline & 2 & 4.7476 & 261 & 0.007898 \\
\hline & 3 & 4.8867 & 254 & 0.001417 \\
\hline & 4 & 4.9139 & 252 & 0.000077 \\
\hline & 5 & 5.0593 & 245 & 0.192269 \\
\hline & 6 & 5.0932 & 243 & 0.000000 \\
\hline & 7 & 5.6427 & 220 & 0.000544 \\
\hline & 8 & 5.664 & 219 & 0.012974 \\
\hline & 9 & 5.7035 & 217 & 0.034977 \\
\hline & 10 & 5.7248 & 217 & 0.000072 \\
\hline & 11 & 5.7686 & 215 & 0.000011 \\
\hline & 12 & 5.7701 & 215 & 0.000236 \\
\hline & 13 & 5.8281 & 213 & 0.020703 \\
\hline & 14 & 5.8367 & 212 & 0.000036 \\
\hline & 15 & 5.8391 & 212 & 0.001040 \\
\hline & 16 & 5.889 & 211 & 0.240317 \\
\hline & 17 & 5.9814 & 207 & 0.561300 \\
\hline & 18 & 6.0356 & 205 & 0.237109 \\
\hline & \#Ex.State & $\mathrm{eV}$ & $\mathrm{nm}$ & $\mathrm{f}$ \\
\hline \multirow[t]{17}{*}{$n=1$} & 1 & 4.6993 & 264 & 0.012195 \\
\hline & 2 & 4.8671 & 255 & 0.000006 \\
\hline & 3 & 4.9218 & 252 & 0.001447 \\
\hline & 4 & 4.9827 & 249 & 0.000076 \\
\hline & 5 & 4.9842 & 249 & 0.224120 \\
\hline & 6 & 5.1258 & 242 & 0.000000 \\
\hline & 7 & 5.6 & 221 & 0.000457 \\
\hline & 8 & 5.7204 & 217 & 0.121659 \\
\hline & 9 & 5.7219 & 217 & 0.008313 \\
\hline & 10 & 5.7365 & 216 & 0.000034 \\
\hline & 11 & 5.8487 & 212 & 0.001105 \\
\hline & 12 & 5.8701 & 211 & 0.098948 \\
\hline & 13 & 5.9562 & 208 & 0.462607 \\
\hline & 14 & 5.9975 & 207 & 0.152293 \\
\hline & 15 & 6.0035 & 207 & 0.000203 \\
\hline & 16 & 6.0151 & 206 & 0.000026 \\
\hline & 17 & 6.0378 & 205 & 0.150654 \\
\hline
\end{tabular}




\begin{tabular}{|c|c|c|c|c|}
\hline & \#Ex.State & $\mathrm{eV}$ & $\mathrm{nm}$ & $f$ \\
\hline \multirow[t]{15}{*}{$\mathrm{n}=2$} & 1 & 4.6666 & 266 & 0.014770 \\
\hline & 2 & 4.932 & 251 & 0.124609 \\
\hline & 3 & 4.9409 & 251 & 0.103395 \\
\hline & 4 & 4.9456 & 251 & 0.011610 \\
\hline & 5 & 5.1437 & 241 & 0.000044 \\
\hline & 6 & 5.1465 & 241 & 0.000022 \\
\hline & 7 & 5.5419 & 224 & 0.000231 \\
\hline & 8 & 5.6676 & 219 & 0.001001 \\
\hline & 9 & 5.7374 & 216 & 0.197619 \\
\hline & 10 & 5.7442 & 216 & 0.015675 \\
\hline & 11 & 5.8585 & 212 & 0.001120 \\
\hline & 12 & 5.9526 & 208 & 0.126528 \\
\hline & 13 & 5.9798 & 207 & 0.541288 \\
\hline & 14 & 6.1102 & 203 & 0.001753 \\
\hline & \#Ex.State & $\mathrm{eV}$ & $\mathrm{nm}$ & $f$ \\
\hline \multirow[t]{14}{*}{$n=3$} & 1 & 4.618 & 268 & 0.018655 \\
\hline & 2 & 4.8633 & 255 & 0.244631 \\
\hline & 3 & 4.9151 & 252 & 0.016894 \\
\hline & 4 & 4.9656 & 250 & 0.001527 \\
\hline & 5 & 5.1459 & 241 & 0.000054 \\
\hline & 6 & 5.1637 & 240 & 0.000015 \\
\hline & 7 & 5.5236 & 224 & 0.000093 \\
\hline & 8 & 5.6513 & 219 & 0.000042 \\
\hline & 9 & 5.7187 & 217 & 0.232980 \\
\hline & 10 & 5.774 & 215 & 0.000108 \\
\hline & 11 & 5.8861 & 211 & 0.001205 \\
\hline & 12 & 5.9719 & 208 & 0.556823 \\
\hline & 13 & 6.0049 & 206 & 0.086070 \\
\hline & \#Ex.State & $\mathrm{eV}$ & $\mathrm{nm}$ & $f$ \\
\hline \multirow[t]{8}{*}{$n=4$} & 1 & 4.5998 & 270 & 0.019812 \\
\hline & 2 & 4.84 & 256 & 0.264681 \\
\hline & 3 & 4.9457 & 251 & 0.004026 \\
\hline & 4 & 4.9694 & 249 & 0.001500 \\
\hline & 5 & 5.1658 & 240 & 0.000036 \\
\hline & 6 & 5.2135 & 238 & 0.000030 \\
\hline & 7 & 5.4624 & 227 & 0.000176 \\
\hline & 8 & 5.5914 & 222 & 0.000711 \\
\hline
\end{tabular}




\begin{tabular}{|c|c|c|c|c|}
\hline & 9 & 5.7128 & 217 & 0.237021 \\
\hline & 10 & 5.7675 & 215 & 0.000998 \\
\hline & 11 & 5.8792 & 211 & 0.001988 \\
\hline & 12 & 5.9723 & 208 & 0.563647 \\
\hline & 13 & 6.0233 & 206 & 0.065507 \\
\hline & \#Ex.State & $\mathrm{eV}$ & $\mathrm{nm}$ & $f$ \\
\hline \multirow[t]{14}{*}{$n=4 m=1$} & 1 & 4.569200 & 271 & 0.03812 \\
\hline & 2 & 4.875800 & 254 & 0.169672 \\
\hline & 3 & 4.931000 & 251 & 0.072587 \\
\hline & 4 & 5.048900 & 246 & 0.001901 \\
\hline & 5 & 5.178500 & 239 & 0.000111 \\
\hline & 6 & 5.222700 & 237 & 0.000547 \\
\hline & 7 & 5.517100 & 225 & 0.000331 \\
\hline & 8 & 5.690100 & 218 & 0.004855 \\
\hline & 9 & 5.728400 & 216 & 0.240664 \\
\hline & 10 & 5.773500 & 215 & 0.00122 \\
\hline & 11 & 5.948000 & 208 & 0.487359 \\
\hline & 12 & 5.969300 & 208 & 0.004382 \\
\hline & 13 & 6.011900 & 206 & 0.134144 \\
\hline & \#Ex.State & $\mathrm{eV}$ & $\mathrm{nm}$ & $f$ \\
\hline \multirow[t]{13}{*}{$n=4 m=2$} & 1 & 4.534100 & 273 & 0.038209 \\
\hline & 2 & 4.8854 & 254 & 0.050025 \\
\hline & 3 & 4.957 & 250 & 0.17861 \\
\hline & 4 & 5.0642 & 245 & 0.00188 \\
\hline & 5 & 5.1611 & 240 & 0.000124 \\
\hline & 6 & 5.2332 & 237 & 0.001351 \\
\hline & 7 & 5.5321 & 224 & 0.00018 \\
\hline & 8 & 5.6945 & 218 & 0.023456 \\
\hline & 9 & 5.7288 & 216 & 7.98E-05 \\
\hline & 10 & 5.751 & 216 & 0.277583 \\
\hline & 11 & 5.8992 & 210 & 0.029415 \\
\hline & 12 & 5.902 & 210 & 0.43317 \\
\hline & 13 & 6.0062 & 206 & 0.124886 \\
\hline
\end{tabular}




\section{Experimental oscillator strength:}

To calculate the oscillator strength for each transition from the experimental optical absorption spectra of gallic acid at different $\mathrm{pH}$, the following equation was used ${ }^{1,2}$ :

$$
f=2303\left(\frac{m c^{2}}{\pi e^{2} N_{A}}\right) \int \varepsilon(v) d v=4.318 \cdot 10^{-9} \int \varepsilon(v) d v
$$

The constant part $4.314 \cdot 10^{-9}$ is in the units $\left(\mathrm{mol} \mathrm{cm}^{2} \mathrm{~L}^{-1}\right)$. The integral is in units of $\left(\mathrm{L} \mathrm{mol}^{-1} \mathrm{~cm}^{-2}\right)$. The oscillator strength $f$ is dimensionless.

$f$ - oscillator strength

$\mathrm{m}-$ mass of an electron, $9.1094 * 10^{-28} \mathrm{~g}$

c - light speed, $2.9978 * 10^{8} \mathrm{~m} / \mathrm{s}$

$\mathrm{N}_{\mathrm{a}}$ - Avogadro number, $6.022 * 10^{23} \mathrm{~mol}^{-1}$

e - elementary charge, $1.602 * 10^{-19}$ coulombs

$\varepsilon-$ molar absorption coefficient $\left(\mathrm{L} \mathrm{M}^{-1} \mathrm{~cm}^{-1}\right)$

$\mathrm{v}$ - wave number $\left(\mathrm{cm}^{-1}\right)$

To obtain the curve fit of the spectra the Gaussian transformation was applied. These allowed us to calculate Integrals and area under the curve for each peak.

Table S5. Experimental and Theoretical peak positions $(\lambda, \mathrm{nm})$ and oscillator strength (f) of optical absorption spectra at different $\mathrm{pH}$.

\begin{tabular}{|c|c|c|c|c|c|}
\hline & & \multicolumn{2}{|c|}{ Experiment } & \multicolumn{2}{|c|}{ Theory } \\
\hline & Band & $\lambda, \mathrm{nm}$ & $f$ & $\lambda, \mathrm{nm}$ & $f$ \\
\hline \multirow[t]{3}{*}{$\mathrm{pH}=2$} & $\mathrm{~A}$ & 295 & 0.01599 & 299 & 0.06506 \\
\hline & $\mathrm{B}$ & 271 & 0.20242 & 278 & 0.29652 \\
\hline & $\mathrm{C}$ & 215 & 0.59749 & $\begin{array}{l}222 \\
211 \\
209 \\
\end{array}$ & $\begin{array}{l}0.273914 \\
0.448011 \\
0.010520 \\
\end{array}$ \\
\hline \multirow[t]{3}{*}{$\mathrm{pH}=6$} & A & 287 & 0.01066 & 273 & 0.03821 \\
\hline & $\mathrm{B}$ & 259 & 0.17083 & 250 & 0.17861 \\
\hline & $\mathrm{C}$ & 211 & 0.68193 & $\begin{array}{l}216 \\
210 \\
206\end{array}$ & $\begin{array}{l}0.27758 \\
0.43317 \\
0.12489\end{array}$ \\
\hline
\end{tabular}

(1) Perkampus, H.-H. UV-VIS Spectroscopy and Its Applications; 1992.

(2) Belay, A. Measurement of Integrated Absorption Cross-Section, Oscillator Strength and Number Density of Caffeine in Coffee Beans by Integrated Absorption Coefficient Technique. Food Chemistry 2010, 121 (2), 585-590. https://doi.org/10.1016/j.foodchem.2009.12.052. 